\title{
ARMAZENAMENTO DE TRICHOGRAMMA PRETIOSUM RILEY (HYMENOPTERA: TRICHOGRAMMATIDAE) EM BAIXAS TEMPERATURAS
}

\author{
S.M.M. Rodrigues ${ }^{1}$; M.V. Sampaio ${ }^{2}$
}

${ }^{1}$ Embrapa Algodão, Núcleo de PD \& I do Mato Grosso, Av. dos Jacaradás, 2639, Setor Residencial, CEP 78550-003, Sinop, MT, Brasil. E-mail: sandra@cnpa.embrapa.br

\section{RESUMO}

\begin{abstract}
Avaliou-se o efeito do armazenamento em baixa temperatura de pupas de Trichogrammapretiosum, em ovos de Sitotroga cerealella, sobre a emergência e a viabilidade reprodutiva do parasitoide. Os testes foram conduzidos em câmara climática a 5,8 e $10^{\circ} \mathrm{C}, 70 \%$ UR, escotofase constante, com 10 tratamentos (testemunha, 4, 6, 8, 10, 12, 14, 16, 18 e 20 dias de armazenamento) e 10 repetições. Foram distribuídos 1.000 ovos contendo pupas em tubos de ensaio $(10 \times 3 \mathrm{~cm}), 100$ foram mantidos a $25^{\circ} \mathrm{C}, 70 \%$ UR e 14 horas de fotofase (testemunha) e os demais armazenados. Após cada período de armazenamento, 100 ovos foram transferidos para $25^{\circ} \mathrm{C}$ e avaliados quanto à emergência e funções reprodutivas. A emergência de T. pretiosum não foi influenciada pelo período de armazenamento nas três temperaturas (Tukey $\mathrm{P} \leq 0,05$ ) e as menores taxas de emergência foram de $85,6 \%, 84,5 \%$ e $77,6 \%$, respectivamente para os parasitoides armazenados a 5, 8 e $10^{\circ} \mathrm{C}$. Não houve perda da viabilidade reprodutiva dos parasitoides provenientes da estocagem nas temperaturas avaliadas, pois todas as fêmeas acasaladas produziram indivíduos de ambos os sexos. Estes resultados auxiliarão no planejamento da criação massal, embalagem e transporte de T. pretiosum do laboratório para o local de liberação.
\end{abstract}

PALAVRAS-CHAVE: Parasitoide de ovos, estocagem, criação massal, controle biológico.

\begin{abstract}
COLD STORAGE OF TRICHOGRAMMA PRETIOSUM RILEY (HYMENOPTERA: TRICHOGRAMMATIDAE). The objective of this work was to evaluate the effect of storage at low temperature of Trichogramma pretiosum pupae in eggs of Sitotroga cerealella on the emergence and reproduction rates of the parasitoid. The trials were conducted in a climatic chamber at 5 , 8 and $10^{\circ}$ C, 70\% R.H., constant scotophase, with 10 treatments: 4, 6, 8, 10, 12, 14, 16, 18, 20 days of storage and a control not kept in storage, all in 10 replicates. A total of 1,000 eggs containing pupae were distributed in glass tubes $(10 \times 3 \mathrm{~cm}), 100$ of which were kept at $25^{\circ} \mathrm{C}, 70 \% \mathrm{RH}$ and $14 \mathrm{~h}$ photophase (control), while the remaining ones were stored. After each storage period 100 eggs were transferred to $25^{\circ} \mathrm{C}$ and the emergence and reproductive capacity were observed. The T. pretiosum emergence rate was not affected by the storage time at 3 temperatures (Tukey $\mathrm{P} \leq 0.05$ ) and the lowest emergence rates were $85.6 \%, 84.5 \%$ and $77.6 \%$, respectively for parasitoid storage at 5,8 and $10^{\circ} \mathrm{C}$. The reproductive capacity of $\mathrm{T}$. pretiosum was not affected after the exposure periods in the temperatures evaluated. This result will aid in the planning of mass production, package and transport of T. pretiosum to the place of release.
\end{abstract}

KEY WORDS: Parasitoid, cold storage, mass rearing, biological control.

\section{INTRODUÇÃO}

Os parasitoides do gênero Trichogramma têm sido usados com sucesso como agente de controle biológico contra insetos-praga da ordem Lepidoptera em diversos sistemas agrícolas (HASSAN; ZHANG, 2001). A liberação de Trichogramma é ainda limitada na América do Sul, principalmente, devido ao alto custo na produção comercial dos parasitoides e pelo uso intensivo de inseticidas, mesmo assim, a área estimada com a utilização de Trichogramma é de 1,2 milhões de hectares. A Colômbia se destaca com uma área com liberação inundativa periódica de Trichogramma de aproximadamente 200.000 hectares (LENTEREN; BuENo, 2002). No Brasil, esse parasitoide tem sido utilizado em algodoeiro, cana-de-açúcar,

${ }^{2}$ Universidade Federal de Uberlândia, Istituto de Ciências Agrárias, Uberlândia, MG, Brasil. 
hortaliças, milho, soja e tomateiro (PARRA; ZuCCHI, 2004).

O controle biológico aumentativo, no qual são feitas liberações periódicas de elevado número de inimigos naturais, é utilizado comercialmente em vários sistemas agrícolas em diversos países. Estima-se que a área total mundial sob controle bio-lógico aumentativo seja de cerca de 32 milhões de hectares, incluindo condições de campo e de cultivos protegidos. Para a aplicação do controle biológico aumentativo, é necessária a criação massal dos inimigos naturais, o que vem ocorrendo em laboratórios comerciais distribuídos em todo o mundo (Lenteren, 2000; LeNTEREN; Bueno, 2002). Os avanços nas áreas de produção massal, armazenamento, envio e liberação de inimigos naturais, têm reduzido os custos de produção, resultando, também, em um produto de melhor qualidade (LENTEREN; TOMMAZINII, 1999).

A eficiência docontrole biológicoé dependente da presença dos parasitoides nomomento em quea praga inicia a colonização das plantas e em uma proporção adequada de parasitoide: hospedeiro(TREMBLAY, 1994). Entre as condições necessárias para que haja sucesso no controle biológico estão o transporte, métodos de liberação quenão comprometam a eficiência doagente de controle e a disponibilidade de armazenamento eficiente (LENTEREN, 2000). Uma vez que a necessidade deuso dos inimigos naturais apresenta uma demanda flutuante é desejável estocá-los por determinados períodos sem que ocorra a perda de sua viabilidade (BRADLEY etal., 2004). A estocagem de insetos em baixa temperatura é uma parte importante do processo de criação massal para uso no controle de pragas tanto em cultivos abertos como em casa-de-vegetação. Muitos predadores e parasitoides podem ser armazenados normalmente como imaturos, mas somente por um tempo bem curto, em temperaturas que variam de 4 a $15^{\circ}$ C. (LENTEREN, 2000).

Uma forma de se garantir quantidade suficiente de parasitoides para o momento de uma liberação nos cultivos é por meio do armazenamento de pré-pupas ou pupas do parasitoide (LEOPOLD, 1998). Porém, Flanders (1938) relatou que himenópteros parasitoides, quando expostos às baixas temperaturas por períodos prolongados, podem não completar o seu desenvolvimento devido a uma quantidade insuficiente de nutrientes, ou por ter os seus órgãos reprodutores afetados pela falta de nutrientes, resultando em machos estéreis, em decorrência da inviabilidade dos espermatozoides. Uma vez que o armazenamento em baixas temperaturas pode reduzir a longevidade, a fecundidade (OzDER, 2008) e a emergência (Geng et al., 2005) de parasitoides do gênero Trichogramma, este trabalho teve por objetivo avaliar o efeito do armazenamento de pupas de Trichogramma pretiosum, em baixas temperaturas, em ovos de Sitotroga cerealella, sobre a emergência e a viabilidade reprodutiva deste parasitoide.

\section{MATERIAL E MÉTODOS}

O experimento foi conduzido na Biofábrica de Inimigos Naturais, pertencente à Fundação Centro Oeste ${ }^{a}$, em Primavera do Leste, MT, com insetos oriundos da criação de manutenção. Cem seções de cartolina contendo 10 ovos cada de $S$. cerealella foram individualizadas em placas de Petri (10 cm de diâmetro), colocadas para serem parasitadas por T. pretiosum e, posteriormente, transferidas para tubos de ensaio $(10 \times 3 \mathrm{~cm})$. Quando foi constatado que o parasitoide estava na fase pupal (ovo escuro), efetuou-se a transferência dos tubos para câmaras climáticas reguladas com temperaturas de 5,8 e $10 \pm 1^{\circ} \mathrm{C}, 70 \pm 10 \%$ UR e com escotofase constante. O lote de cem ovos (10 tubos com 10 ovos parasitados por tubo) que constituíam a testemunha foi colocado em câmara climática a $25 \pm 1^{\circ} \mathrm{C}, 70 \pm 10 \%$ UR e 14 horas de fotofase.

Quatro dias após o início da estocagem, um lote de cem ovos foi retirado de cada câmara climática e transferido para outra com $25 \pm 1^{\circ} \mathrm{C}, 70 \pm 10 \%$ UR e 14 horas de fotofase, condições que são adequadas para o desenvolvimento do parasitoide. Esse processo foi repetido a cada dois dias, até o $20^{\circ}$ dia de estocagem, e para as três temperaturas avaliadas. Foram formados 10 tratamentos: 0 (testemunha), $4,6,8,10,12,14,16,18$ e 20 dias de armazenamento em três temperaturas, com dez repetições, correspondendo cada a um tubo contendo 10 ovos parasitados.

Foram observadas diariamente a emergência dos parasitoides e a presença de defeitos nos adultos emergidos, com o auxílio de um microscópio estereoscópico. O número de parasitoides emergidos e que apresentaram mortalidade no primeiro dia após a emergência também foi avaliado. Para a avaliação da viabilidade de óvulos e espermatozoides dos parasitoides armazenados, fêmeas e machos oriundos do mesmo período de armazenamento e emergidos no mesmo dia foram acasalados e alimentados com solução de mel a $20 \%$. Posteriormente, cada fêmea acasalada foi colocada em contato com uma seção de cartolina contendo 10 ovos do hospedeiro, por um período de 24 horas. Em seguida, os ovos foram colocados em câmara climática $\left(25 \pm 1^{\circ} \mathrm{C}, 70 \pm 10 \%\right.$ UR e 14 horas de fotofase) onde os parasitoides se desenvolveram, até a emergência da prole. $\mathrm{O}$ efeito da temperatura sobre os órgãos reprodutores dos parasitoides emergidos, após a exposição das pupas a 5,8 e $10^{\circ} \mathrm{C}$, foi verificado em casais de mesma idade oriundos dos seguintes tratamentos: testemunha, 4,6 e 8

*Extinta em 2006. 
dias de armazenamento. Nos demais tratamentos não foi possível formar casais com a mesma idade.

O delineamento estatístico utilizado foi o inteiramente casualizado, no esquema fatorial 3 x 10 ( temperatura $\mathrm{x}$ período de armazenamento), com 10 repetições. Os dados foram submetidos à análise de variância e as médias comparadas pelo teste de Tukey, ao nível de 5\% de significância.

\section{RESULTADOS E DISCUSSÃO}

Nãohouveinteração significativa entre a temperatura e o tempo de armazenamento na emergência dos parasitoides. O período de armazenamento não influenciou a emergência de T. pretiosum dentro das temperaturas de 5,8 e $10^{\circ} \mathrm{C}$. No entanto, houve diferença estatística para a emergência do parasitoidenas diferentes temperaturas. As porcentagens deadultos emergidos nas temperaturas de $5^{\circ} \mathrm{C}(95,8 \%)$ e $8^{\circ} \mathrm{C}$ $(95,9 \%)$ foram semelhantes entre si e superiores a de $10^{\circ} \mathrm{C}(84,3 \%)$ (Tabela 1$)$.

Em programas de controle de qualidade de Trichogramma, a viabilidade é considerada adequada quando a emergência de adultos é superior a $90 \%$ (KNUTSON, 1998). Assim, pode-se verificar que, nas temperaturas de 5 e $8^{\circ} \mathrm{C}$, a emergência desse parasitoide, desenvolvido em ovos de $S$. cerealella e armazenado em 5 e $8^{\circ} \mathrm{C}$, atingiu índices superiores ao considerado satisfatório, exceto no período de 10 dias de armazenamento (83,5\%) a $5^{\circ} \mathrm{C}$. Já na temperatura de $10^{\circ} \mathrm{C}$ não se observou taxas superiores a $90 \%$ de emergência, exceto no período de 8 dias de armazenamento $(95,6 \%$ ) (Tabela 1). Portanto, de acordo com a porcentagem de emergência dos adultos, as temperaturas de 5 e $8^{\circ}$ $C$ foram mais adequadas para armazenar as pupas do parasitoide do que a de $10^{\circ} \mathrm{C}$. Também, Geng et al. (2005) consideraram que a temperatura de $10^{\circ}$ $C$ foi inadequada para armazenar o parasitoide $T$. dendrolimi por longos períodos, pois foi a que mais afetou a sua taxa de emergência.

Quanto à razão sexual, não se constatou diferença entre os períodos de armazenamento dentro de cada temperatura (Tabela 2). Neste experimento a razão sexual foi sempre igual ou superior a 0,5 ; este valor se encontra dentro das especificações exigidas pelo IOBC (2002).

A mortalidade no primeiro dia após a emergência é um aspecto importante a ser observado nos adultos emergidos após o processo de armazenamento em baixas temperaturas. A mortalidade precoce indica uma redução na longevidade do parasitoide que pode torná-lo não efetivo no controle da praga. A interação da temperatura com o período de armazenamento foi significativa para a mortalidade no primeiro dia após a emergência. Essa mortalidade foi superior em $8^{\circ} \mathrm{C}$ do que em $5^{\circ} \mathrm{C}$, com médias de $40,5 \pm 3,15 \%$ e $29,4 \pm 2,84 \%$, respectivamente. Já a mortalidade no primeiro dia após a emergência a $10^{\circ} \mathrm{C}$ não apresentou diferença entre as temperaturas de 5 e $8^{\circ} \mathrm{C}$ (Tabela 3). Essa mortalidade foi inferior em $5^{\circ} \mathrm{C}$ do que em $8^{\circ} \mathrm{C}$ após 12,16 e 20 dias, e inferior a $8^{\circ} \mathrm{C}$ após 16 e 20 dias de armazenamento. Já a mortalidade no primeiro dia após a emergência foi similar para as temperaturas $8^{\circ} \mathrm{C}$ e $10^{\circ} \mathrm{C}$ em todos os períodos de armazenamento avaliados (Tabela 3). Essa mortalidade não foi afetada pelo período de armazenamento a $5^{\circ} \mathrm{C}$, tendo apresentado média de $29,4 \pm 2,84 \%$ (Fig. 1).

Tabela 1 - Emergência (\%) de Trichogramma pretiosum desenvolvido em ovos de Sitotroga cerealella e armazenado com escotofase constante por diferentes períodos e temperaturas, e transferido posteriormente para $25^{\circ} \mathrm{C}, 70 \pm 10 \%$ UR e 14 horas de fotofase.

\begin{tabular}{cccc}
\hline & \multicolumn{3}{c}{ Temperatura $\left({ }^{\circ} \mathrm{C}\right)$} \\
\cline { 2 - 4 } Período de armazenamento $(\mathrm{d})$ & 8 & 10 \\
\cline { 2 - 4 } & 5 & $(\bar{X} \pm \mathrm{EP})^{1}$ & $79,8 \pm 5,97$ \\
\cline { 2 - 4 } & $95,0 \pm 5,00$ & $95,4 \pm 3,43$ & $77,6 \pm 6,13$ \\
4 (testemunha) & $98,2 \pm 1,79$ & $96,7 \pm 3,33$ & $88,3 \pm 5,67$ \\
6 & $95,8 \pm 4,17$ & $100,0 \pm 0,00$ & $95,6 \pm 2,94$ \\
8 & $100,0 \pm 0,00$ & $92,1 \pm 3,44$ & $87,8 \pm 4,89$ \\
10 & $83,5 \pm 8,87$ & $96,3 \pm 2,46$ & $85,4 \pm 7,83$ \\
14 & $97,9 \pm 2,08$ & $94,3 \pm 4,05$ & $80,3 \pm 5,58$ \\
16 & $100,0 \pm 0,00$ & $100,0 \pm 0,00$ & $88,8 \pm 5,64$ \\
20 & $100,0 \pm 0,00$ & $94,7 \pm 3,69$ & $80,7 \pm 7,21$ \\
\hline Média Geral ${ }^{2}$ & $97,5 \pm 2,50$ & $95,0 \pm 5,00$ & $79,0 \pm 6,64$ \\
\hline
\end{tabular}

${ }^{1}$ Médias na coluna são semelhantes entre si pela diferença mínima significativa (DMS) $(\mathrm{P}=0,05)$.

${ }^{2}$ Médias seguidas da mesma letra na linha, não diferem entre si, pelo teste de Tukey $(\mathrm{P}=0,05)$. 
Tabela 2 - Razão sexual de Trichogramma pretiosum desenvolvido em ovos de Sitotroga cerealella e armazenado com escotofase constante por diferentes períodos e temperaturas, e transferido posteriormente para $25^{\circ} \mathrm{C}, 70 \pm 10 \%$ UR e 14 horas de fotofase.

\begin{tabular}{cccc}
\hline & \multicolumn{3}{c}{ Temperatura $\left({ }^{\circ} \mathrm{C}\right)$} \\
\cline { 2 - 4 } Período de armazenamento $(\mathrm{d})$ & 5 & 8 & 10 \\
\cline { 2 - 4 } & $(\overline{\mathrm{X}} \pm \mathrm{EP})^{1}$ & $(\overline{\mathrm{X}} \pm \mathrm{EP})^{1}$ & $0,6 \pm 0,07$ \\
\hline 0 (testemunha) & $0,8 \pm 0,06$ & $0,7 \pm 0,08$ & $0,6 \pm 0,07$ \\
4 & $0,5 \pm 0,10$ & $0,6 \pm 0,05$ & $0,7 \pm 0,05$ \\
6 & $0,6 \pm 0,08$ & $0,7 \pm 0,08$ & $0,7 \pm 0,07$ \\
8 & $0,5 \pm 0,06$ & $0,7 \pm 0,04$ & $0,8 \pm 0,07$ \\
10 & $0,5 \pm 0,06$ & $0,7 \pm 0,05$ & $0,9 \pm 0,06$ \\
12 & $0,6 \pm 0,10$ & $0,8 \pm 0,06$ & $0,7 \pm 0,08$ \\
14 & $0,7 \pm 0,08$ & $0,7 \pm 0,05$ & $0,8 \pm 0,04$ \\
16 & $0,6 \pm 0,03$ & $0,8 \pm 0,04$ & $0,7 \pm 0,06$ \\
20 & $0,8 \pm 0,06$ & $0,7 \pm 0,07$ & $0,7 \pm 0,07$ \\
\hline
\end{tabular}

${ }^{1}$ Médias na coluna são semelhantes entre si pela diferença mínima significativa (DMS) $(\mathrm{P}=0,05)$

Tabela 3 - Mortalidade (\%) no primeiro dia de vida de Trichogramma pretiosum desenvolvido em ovos de Sitotroga cerealella e armazenado com escotofase constante por diferentes períodos e temperaturas, e transferido posteriormente para $25^{\circ} \mathrm{C}, 70 \pm 10 \%$ UR e 14 horas de fotofase.

\begin{tabular}{cccc}
\hline & \multicolumn{3}{c}{ Temperatura $\left({ }^{\circ} \mathrm{C}\right)$} \\
\cline { 2 - 4 } Período de armazenamento $(\mathrm{d})$ & \multicolumn{3}{c}{10} \\
\cline { 2 - 4 } & 5 & $(\overline{\mathrm{X}} \pm \mathrm{EP})^{1}$ & $(\bar{X} \pm \mathrm{EP})^{1}$ \\
\hline 0 (testemunha) & $16,0 \pm 6,35 \mathrm{a}$ & $36,6 \pm 12,13 \mathrm{a}$ & $10,1 \pm 10,20 \mathrm{a}$ \\
4 & $16,3 \pm 5,94 \mathrm{a}$ & $10,5 \pm 4,87 \mathrm{a}$ & $32,0 \pm 7,24 \mathrm{a}$ \\
6 & $16,5 \pm 9,13 \mathrm{a}$ & $29,1 \pm 10,12 \mathrm{a}$ & $30,0 \pm 9,83 \mathrm{a}$ \\
10 & $45,8 \pm 7,66 \mathrm{a}$ & $21,8 \pm 5,64 \mathrm{a}$ & $24,0 \pm 5,76 \mathrm{a}$ \\
14 & $41,3 \pm 5,56 \mathrm{a}$ & $23,1 \pm 5,74 \mathrm{a}$ & $49,0 \pm 7,27 \mathrm{ab}$ \\
16 & $26,4 \pm 6,57 \mathrm{~b}$ & $56,8 \pm 9,74 \mathrm{a}$ & $39,0 \pm 8,55 \mathrm{a}$ \\
20 & $48,3 \pm 13,26 \mathrm{a}$ & $47,8 \pm 7,23 \mathrm{a}$ & $56,2 \pm 9,35 \mathrm{a}$ \\
& $14,9 \pm 4,55 \mathrm{~b}$ & $55,6 \pm 9,73 \mathrm{a}$ & $50,8 \pm 9,49 \mathrm{a}$
\end{tabular}

${ }^{1}$ Médias seguidas da mesma letra minúscula na linha, não diferem entre si, pelo teste de Tukey $(\mathrm{P}=0,05)$.

Já nas temperaturas de 8 e $10^{\circ} \mathrm{C}$ observou-se que a mortalidade no primeiro dia, após a emergência, aumentou linearmente em função do aumento do período dearmazenamento, se ajustando a uma equação de primeiro grau (Figs. 2 e 3). Observou-se que a $8^{\circ} \mathrm{C}$ a mortalidade no primeiro dia de vida atingiu 70,5\% após vinte dias de armazenamento (Fig. 2), e a $10^{\circ} \mathrm{C}$ essa mortalidade foi de $50 \%$ com 20 dias de armazenamento (Fig. 3). Embora o armazenamento a $8^{\circ} \mathrm{C}$ não tenha comprometido a emergência de T. pretiosum, provocou uma alta porcentagem de mortalidade no primeiro dia de vida nos adultos do parasitoide, indicando uma redução no número de parasitoides viáveis. Desta forma, somente no armazenamento a $5^{\circ} \mathrm{C}$ foram obtidas emergência alta e mortalidade no primeiro dia de vida baixa após 20 dias de armazenamento.

O parasitismo efetuado pelas fêmeas oriundas dos três períodos de armazenamento (4, 6 e 8 dias) foi de $100 \%$ e, em decorrência do baixo número de repetições (casais), não foi efetuada análise estatística. Não foi constatada perda da capacidade reprodutiva nos machos de T. pretiosum provenientes da estocagem a 5,8 e $10^{\circ} \mathrm{C}$, uma vez que todas as fêmeas acasaladas produziram indivíduos de ambos os sexos.

Segundo Flanders (1938), himenópteros parasitoides expostos às baixas temperaturas por períodos prolongados podem não completar o seu desenvolvimento devido a uma quantidade insuficiente de 
nutrientes, ou podem ter os seus órgãos reprodutores afetados pela falta de nutrientes, podendo o macho tornar-se estéril em decorrência da inviabilidade dos espermatozoides.

Foram observados defeitos nos adultos emergidos, como asas coladas ou atrofiadas, variando de 0,2 a $0,8 \%$, porém não se detectou diferença estatística pelo teste de Tukey $(\mathrm{P}=0,05)$ entre os períodos de armazenamento nas três temperaturas avaliadas.
As informações obtidas neste trabalho auxiliarão no estabelecimento de um programa de armazenagem de pupas de T. pretiosum, que é um item fundamental em um programa de controle biológico. Também, será possível planejar-se a produção e a liberação em campo de T. pretiosum no momento e proporção adequados visando controlar as populações de lepidópteros-praga em lavouras de algodoeiro.

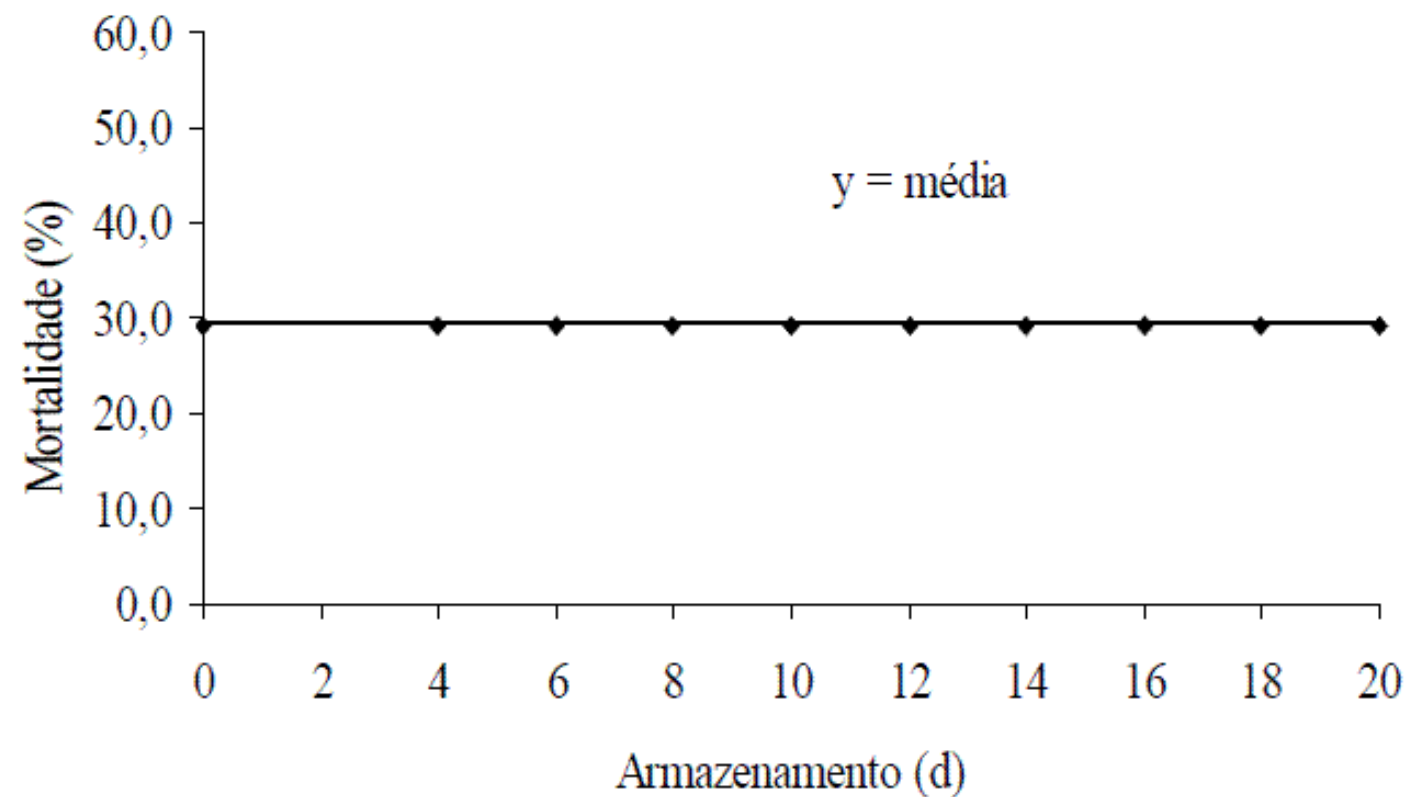

Fig. 1 - Mortalidade no primeiro dia de vida (\%) de Trichogramma pretiosum desenvolvido em ovos de Sitotroga cerealella e armazenado com escotofase constante por diferentes períodos a $5^{\circ} \mathrm{C} \pm 1^{\circ} \mathrm{C}, 70 \pm 10 \%$ UR e transferido posteriormente para $25^{\circ} \mathrm{C}, 70 \pm 10 \%$ UR e 14 horas de fotofase.



Fig. 2 - Mortalidade no primeiro dia de vida (\%) de Trichogramma pretiosum desenvolvido em ovos de Sitotroga cerealella e armazenado com escotofase constante por diferentes períodos a $8^{\circ} \mathrm{C} \pm 1^{\circ} \mathrm{C}, 70 \pm 10 \%$ UR e transferido posteriormente para $25^{\circ} \mathrm{C}, 70 \pm 10 \%$ UR e 14 horas de fotofase. 


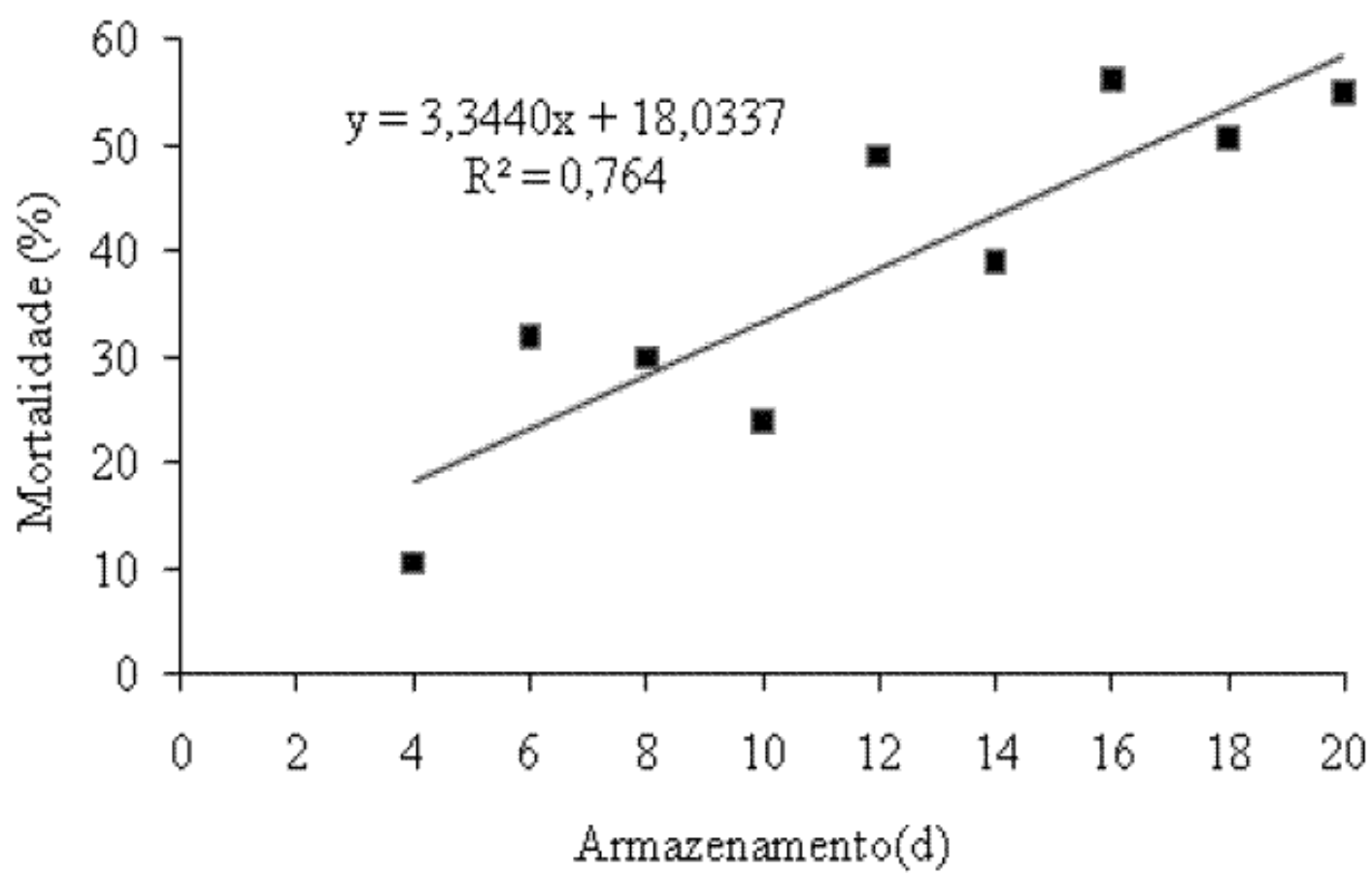

Fig. 3 - Mortalidade no primeiro dia de vida (\%) de Trichogramma pretiosum desenvolvido em ovos de Sitotroga cerealella e armazenado com escotofase constante por diferentes períodos a $10^{\circ} \mathrm{C} \pm 1^{\circ} \mathrm{C}, 70 \pm 10 \%$ UR e transferido posteriormente para $25^{\circ} \mathrm{C}, 70 \pm 10 \%$ UR e 14 horas de fotofase.

\section{CONCLUSÕES}

De acordo com os resultados obtidos pode-se inferir que é possível armazenar pupas de T. pretiosum por até 20 dias a $5^{\circ} \mathrm{C}$ com escotofase constante.

As temperaturas de $8^{\circ} \mathrm{Ce} 10^{\circ} \mathrm{C}$ são inadequadas para armazenar os parasitoides. A $8^{\circ} \mathrm{C}$ e $10^{\circ} \mathrm{C}$ há mortalidade crescente no primeiro dia de vida dos parasitoides adultos e a $10^{\circ} \mathrm{C}$ a emergência é inferior ao determinado nas normas de controle de qualidade para parasitoides do gênero Trichogramma.

\section{AGRADECIMENTOS}

Ao Fundo de Apoio à Cultura do Algodão (FACUAL) por financiar esta pesquisa. À Dalt-Cléa Evangelista Bonfim, laboratorista da Fundação Centro Oeste (FCO), pelo apoio na execução desta pesquisa.

\section{REFERÊNCIAS}

BRADLEY, J. R.; THOMSON, L. J.; HOFFMANN, A. A. Effects of cold storage on field and laboratory performance of Trichogramma carverae (Himenoptera: Trichogrammatidae) and the response of three Trichogramma spp. (T. carverae, T. nr. brassicae and T. funiculatum) to cold. Journal Economic Entomology, v.97, n.2, p.213-221, 2004.
FLANDERS, S.E. The effect of cold storage on the reproduction of parasitic hymenoptera. Journal Economic Entomology, v.31, p.633-634, 1938.

GENG, J.H. ; SHEN, Z.R.; LI, Z.X.; ZHANG, F. Optimal stage and temperature for cold storage of Trichogramma dendrolimi reared on Antheraea pernyi eggs. Acta Entomologica Sinica, v.48, n.6, p.903-909, 2005.

HASSAN, S.A.; ZHANG, W.Q. Variability in quality of Trichogramma brassicae (Hymenoptera: Trichogrammatidae) from commercial suppliers in Germany. Biological Control, v.22, n.2, p.115-121, 2001.

\section{INTERNATIONAL ORGANIZATION FOR BIOLOGICAL} CONTROL. Quality control guidelines for natural enemies. 2002. 31p. Disponível em: <http:/ / users.ugent. be/ padclerc/AMRQC/images/guidelines.pdf $>$. Acesso em: 10 mai. 2005.

KNUTSON, A. The Trichogramma manual, A guide to the use of Trichogramma for biological control with special reference to augmentative releases for control of bollworm and budworm in cotton. Texas: Texas A \&M University, 1998. 42p. (Texas A\&M University: B-6071). Disponível em: <http://insects.tamu.edu/ extension/bulletins/b-6071.html>. Acesso em: 10 mai. 2005.

LENTEREN, J.C.; TOMMASINI, M.G. Mass production, storage, shipment and quality control of natural enemies. In: ALBAJES, R. M.; GULLINO, L.; LENTEREN, J.C.; ELAD, Y. (Ed.). Integrated pest and disease manage- 
ment in greenhouse crops. Dordrecht: Kluwer Academic Publishers, 1999. p.276-294.

LENTEREN J.C. Success in biological control of arthropods by augmentation of natural enemies. In: GURR G.; WRATTEN S. (Ed.). Biological Control: measures of success. Dordrecht: Kluwer Academic Publishers, 2000. chap.3. p.77-103.

LENTEREN, J.C.; BUENO, V.H.P. Augmentative biological control of arthropods in Latin America. Biocontrol, v.48, n.2, p.123-139, 2002.

LEOPOLD, R.A. Cold storage of insects for integrated pest management. In: HALMAN, G.J.; DENLINGER, D.L. (Ed.). Temperature sensitivity in insects and application in integrated pest management. Boulder: Westview Press, 1998. p.235-267. Disponível em: <http:/ / pestdata.ncsu.edu/IPMtext/chap9.pdf>. Acesso em: 20 ago. 2008.

OZDER, N. Effect of cold storage of adult Trichogramma brassicae, T. cacoeciae and T. evanescens (Hym.: Trichogrammatidae). Archives Phytoparasitica Plant Protection, v.41, n.4, p.296-299, 2008.

PARRA, J.R.P.; ZUCCHI, R.A. Trichogramma in Brazil: feasibility of use after twenty years of research. Biological Entomology, v.33, n.3, p.271-281, 2004.

TREMBLAY, E. Management of the host-parasitoid relationships in endophagous Hymenoptera. Bulletin OILB/SROP, v.7, p.285-36, 1994.

Recebido em $11 / 1 / 10$

Aceito em 29/12/10 